\title{
DESIGN OF PRECODING AND EQUALIZATION FOR BROADBAND MIMO TRANSMISSION
}

\author{
Chi Hieu Ta and Stephan Weiss \\ Communications Research Group, School of Electronics and Computer Science \\ University of Southampton, Southampton SO17 1BJ, UK
}

Keywords: Precoding, equalization, broadband MIMO.

\begin{abstract}
We propose a new approach to broadband MIMO precoding and equalisation by the use of a broadband singular value decomposition to decouple the MIMO system matrix into independent subchannels. In a second step, ISI present in the subchannels is eliminated using methods developed for SISO systems. A numerical example is given.
\end{abstract}

\section{Introduction}

Multi-input multi-output (MIMO) systems have attracted much attention recently due to their promise to significantly improve the channel capacity and the link reliability [1]. The key reason for this fact is that in MIMO systems aimed at capacity increases, multiple data streams or signals can be transmitted over the channel simultaneously, which is often referred to as spatial multiplexing.

In order to exploit a MIMO system for transmission with increased capacity, one must eliminate co-channel interference (CCI) between independent data streams as well as eliminate inter-symbol interference (ISI) in case of a frequency selective channel. A considerable amount of research has focussed on the narrowband case, where e.g. [5] has shown that joint precoder and equaliser design can achieve much if deployed on their own, provided that the channel state information (CSI) can be made available to both sides of the transmission link. In the narrowband case the channel matrix can be diagonalised by means of mostly SVD-based operations.

The demand of high transmission rate, however, requires the use of broadband systems which are affected by frequency selective channels, i.e. the link between any receiver and transmitter antenna pair is characterised by an impulse response. Therefore, broadband MIMO systems, in which each sub channel between transmit and receive antennas is considered as a frequency selective fading channel, has drawn great interest of researchers. A method that is often used to remove CCI and ISI in broadband MIMO systems is to introduce redundancy to the data streams. In [8] and [7] optimal solutions to the joint precoder- equalizer design problem for block transmission over single input single output (SISO) frequency selective channels are discussed. The optimality criteria considered are the zero forcing (ZF) and minimum mean square error (MMSE) criteria [8] and the maximization of mutual information [7]. Solu- tions in [8] and [7] are then extended for the MIMO case in [9]. Palomar et al. [3] generalize the results on joint design of linear precoding-equalization to several criteria, classified into Schur-concave and Schur-convex objective functions. In general, there the equalizer matrix is derived as MMSE filter and the precoder matrix is determined through the SVD of the whitened channel.

In this paper, we propose a method for precoding and equalization for point-to-point broadband MIMO channels. Different from block transmission, we utilise in a first step a recently proposed broadband singular value decomposition (BSVD) to eliminate CCI. In a second step, the decoupled SISO channels are precoded and equalised using standard methods such as in [8].

The paper is organised as follows. In Sec. 2, the overall channel and system setup are laid out. Sec. 3 addresses the first step in the proposed design, aiming at CCI cancellation, while Sec. 4 considers the minimisation of ISI. Finally, a numerical example is provided in Sec. 5, while conclusions are drawn in Sec. 6.

In our notation, we use lower- and uppercase boldface font for vector and matrix quantities, respectively. Vector quantities in the z-domain are also denoted as underline variables, e.g. $\underline{X}(z)$. The operator $\{\tilde{\tilde{\mathbf{A}}}(z)$ denotes the parahermitian transpose, i.e. $\tilde{\mathbf{A}}(z)=\mathbf{A}^{\mathrm{H}}\left(z^{-1}\right)$.

\section{Channel Model and System Setup}

\subsection{Spatio-Temporal MIMO System Matrix}

In the following, we assumed a stationary MIMO channel with transfer function $\mathbf{C}(z) \in \mathbb{C}^{R \times T}(z)$, such that

$$
\mathbf{C}(z)=\sum_{l=0}^{L-1} \mathbf{C}[l] z^{-l}
$$

characterises a frequency selective MIMO system with $T$ transmit and $R$ receive antennas. The maximum support length of the channel impulse responses (CIRs) between each pair of transmit and receive antennas is $L$. The matrix $\mathbf{C}_{l} \in \mathbb{C}^{R \times T}$ contains the $l$ th time slice of these CIRs. We further assume that each of the $T$ MIMO inputs emerges from a time multiplex of $J$ input lines. Similarly, each of the $R$ output can be demultiplexed into $J$ signals. The resulting spatio-temporal MIMO system with input $\underline{S}(z) \in \mathbb{C}^{J T}$ and output $\underline{R}(z) \in \mathbb{C}^{J R}$ can be written as $\underline{R}(z)=\mathbf{H}(z) \underline{S}(z)$, whereby the spatio-temporal 
MIMO matrix, by appropriate ordering of $\underline{S}(z)$ and $\underline{R}(z)$, takes the block-pseudo-circulant form

$$
\mathbf{H}(z)=\left[\begin{array}{cccc}
\mathbf{C}_{0}(z) & z^{-1} \mathbf{C}_{P-1}(z) & \cdots & z^{-1} \mathbf{C}_{1}(z) \\
\mathbf{C}_{1}(z) & \mathbf{C}_{0}(z) & \cdots & z^{-1} \mathbf{C}_{2}(z) \\
\vdots & & \ddots & \vdots \\
\mathbf{C}_{P-1}(z) & \mathbf{C}_{P-2}(z) & \cdots & \mathbf{C}_{0}(z)
\end{array}\right]
$$

The matrices $\mathbf{C}_{p}(z), p=0,1, \ldots P-1$, are the $P$ polyphase components of $\mathbf{C}(z)$ such that

$$
\mathbf{C}(z)=\sum_{p=0}^{P-1} \mathbf{C}_{p}\left(z^{P}\right) z^{-p}
$$

or alternatively $\mathbf{C}_{p}(z)=\sum_{n} \mathbf{C}[n P+p] z^{-n}$.

\subsection{Precoder and Equaliser}

Fig. 1 indicates the positions of a precoder $\mathbf{U}(z) \in \mathbb{C}^{P T \times P M}$ and an equaliser $\tilde{\mathbf{B}}(z) \in \mathbb{C}^{P M \times P R}$ in the transmitter and receiver, respectively, whereby $M \leq \min \{T, R\}$. As hinted in Sec. 1, the aim of $\mathbf{U}(z)$ and $\tilde{\mathbf{B}}(z)$ is to diagonalise $\mathbf{H}(z)$, thus eliminating CCI, and to reduce each polynomial on the main diagonal to a scalar element in order to suppress ISI.

In [5], a narrowband system with $P=1$ and $\mathbf{H}(z)=$ $\mathbf{C}[0]=$ constant is addressed. There, the precoder and equalise are not of polynomial form and can be calculated by singular value decomposition (SVD) of $\mathbf{C}[0]$, or a sequence of SVDs in case the received signals cannot be jointly processed, resulting in a diagonalised system that therefore avoids CCI but does not impose ISI because of its narrowband nature.

In a broadband scenario for $P=1$, the $\mathbf{H}(z)=\mathbf{C}(z)$ is an $R \times T$ polynomial matrix of order $L-1$. As the number of polyphase components $P$ is increased, the matrix size increases, but the polynomial order reduces in accordance with the shortening polyphase responses. Once $P=L$ is reached, the polyphase components $\mathbf{C}_{p}(z)$ are constants with no dependency on $z$. However, the block-pseudo-circulant form of (2) means for all $P>L$, the spatio-temporal MIMO system ma$\operatorname{trix} \mathbf{H}(z)$ will be a first order polynomial.

In order to overcome the polynomial order, the block-transmission based systems in [8] for $T=R=1$ and in [9] for arbitrary $T$ and $R$ rely on a time multiplex that is chosen longer than the channel support, i.e. $P=M+L$. As a result, the matrix $\mathbf{H}(z)$ in (2) is a sparse block-pseudo-circulant matrix of
$\mathbf{X}(z)$

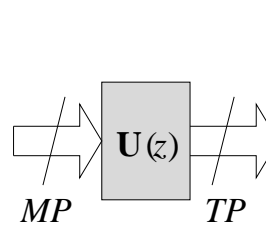

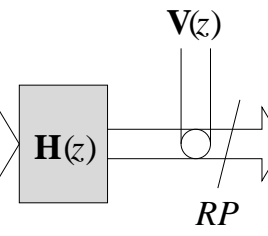

$R P$

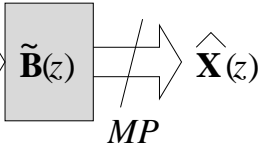

only first order in $z$, as noted earlier. Specifically,

$$
\begin{aligned}
& \mathbf{H}(z)=\mathbf{H}_{0}+z^{-1} \mathbf{H}_{1} \quad \text { with } \\
& \mathbf{H}_{0}=\left[\begin{array}{ccccc}
\mathbf{C}_{0} & 0 & \ldots & \ldots & 0 \\
\vdots & \ddots & & & \\
\mathbf{C}_{L-1} & & \mathbf{C}_{0} & & \\
& \ddots & & \ddots & 0 \\
0 & & \mathbf{C}_{L-1} & \ldots & \mathbf{C}_{0}
\end{array}\right] \\
& \mathbf{H}_{1}=\left[\begin{array}{cccccc}
0 & \ldots & 0 & \mathbf{C}_{L-1} & \ldots & \mathbf{C}_{1} \\
& & & & \ddots & \vdots \\
\vdots & & & & & \mathbf{C}_{L-1} \\
\vdots & & & & & 0 \\
& & & & & \vdots \\
0 & & \ldots & \ldots & & 0
\end{array}\right]
\end{aligned}
$$

Since terms with $z^{-1}$ are only located in the upper right triangle, two methods can be chosen to eliminate the polynomial order of the MIMO system matrix by suppressing $\mathbf{H}_{1}$ such that only a part of $\mathbf{H}_{0}$ in (5) is utilised: (i) transmitting $L-1$ leading zeros in $\underline{S}(z)$, or (ii) discarding the first $L-1$ in $\underline{R}(z)$. Thus eliminating the polynomial nature of $\mathbf{H}(z)$, the precoder and equaliser systems can be selected as non-polynomial matrices whose design can be accomplished using standard linear algebraic methods.

\subsection{Proposed Design}

The approach to MIMO precoding and equalisation proposed in this paper is based on a broadband singular value decomposition (BSVD) of $\mathbf{H}(z)$ as proposed in [2, 6], such that

$$
\mathbf{H}(z)=\mathbf{B}(z)\left[\begin{array}{cc}
\boldsymbol{\Sigma}(z) & \mathbf{0} \\
\mathbf{0} & \mathbf{0}
\end{array}\right] \tilde{\mathbf{U}}(z)
$$

whereby $\boldsymbol{\Sigma}(z)=\operatorname{diag}\left\{\Sigma_{0}(z), \Sigma_{1}(z), \cdots \Sigma_{P M}(z)\right\}$. This permits to take the polynomial nature of the MIMO channel transfer function into account without resorting to block transmission, giving greater flexibility in the choice of $M$ and hence the code rate and subsequently the coding gain. However, the diagonalisation according to (7) only eliminates $\mathrm{CCI}$, while each of the $P M$ subchannels is dispersive and causes ISI.

To cancel ISI, in a second step a precoder and equaliser can be designed for each subchannel, e.g. according to the SISO design discussed in [8]. Thus, block transmission is invoked, but only for small portion of the system design. In addition, the precoder and equaliser design can take the individual properties of each subchannel, such as the SNR, into account.

\section{MIMO System Decomposition Via BSVD}

\subsection{Broadband Singular Value Decomposition}

In the following we characterise the BSVD described in [2,

6 , and the resulting properties of the subchannels $\Sigma_{m}(z)$. A
Fig. 1. MIMO channel $\mathbf{H}(z)$ with precoder $\mathbf{U}(z)$ and equaliser $\tilde{\mathbf{B}}(z)$. 
BSVD can be obtained via two broadband eigenvalue decompositions, whereby a parahermitian matrix $\mathbf{R}_{1}(z)=\mathbf{H}(z) \tilde{\mathbf{H}}(z)$ is decomposed such that

$$
\mathbf{R}_{1}(z)=\mathbf{B}(z) \boldsymbol{\Gamma}_{1}(z) \tilde{\mathbf{B}}(z)
$$

Besides the diagonality of $\boldsymbol{\Gamma}_{1}(z)=\boldsymbol{\Sigma}(z) \tilde{\boldsymbol{\Sigma}}(z)$, we demand paraunitarity of $\mathbf{B}(z)$ such that $\mathbf{B}(z) \tilde{\mathbf{B}}(z)=\tilde{\mathbf{B}}(z) \mathbf{B}(z)=\mathbf{I}$ and spectral majorisation of $\boldsymbol{\Gamma}_{1}(z)$ [11] such that the diagonal elements of

$$
\boldsymbol{\Gamma}_{1}(z)=\operatorname{diag}\left\{\Gamma_{0}(z), \Gamma_{1}(z), \ldots \Gamma_{K-1}(z)\right\}
$$

are ordered according to

$$
\Gamma_{k}\left(e^{j \Omega}\right) \geq \Gamma_{k+1}\left(e^{j \Omega}\right) \quad \forall \Omega \quad \text { and } \quad k=0,1, \ldots, K-2 \text {, }
$$

similar to the ranking of the singular values in a singular value decomposition. Note that paraunitarity or losslessness of $\mathbf{B}(z)$ conserves power, i.e. $\left.\operatorname{tr}\left\{\boldsymbol{\Gamma}_{1}(z)\right\}\right|_{z=0}=\left.\operatorname{tr}\left\{\mathbf{R}_{1}(z)\right\}\right|_{z=0}$.

In a similar operation, $\mathbf{U}(z)$ can be obtained via broadband EVD of $\mathbf{R}_{2}(z)=\tilde{\mathbf{H}}(z) \mathbf{H}(z)$.

\subsection{Sequential Best Rotation Algorithm}

In order to achieve the factorisation in (8) fulfilling spectral majorisation according to (10), we use the second order sequential best rotation (SBR2) algorithm [2, 6], whose steps we briefly review below.

SBR2 is an iterative broadband eigenvalue decomposition technique based on second order statistics only and can be seen as a generalisation of the Jacobi algorithm. The decomposition after $L$ iterations is based on a paraunitary matrix $\mathbf{B}_{L}(z)$,

$$
\mathbf{B}_{L}(z)=\prod_{i=0}^{L} \mathbf{Q}_{i} \mathbf{\Lambda}_{i}(z)
$$

whereby $\mathbf{Q}_{i}$ is a Givens rotation and the matrix $\boldsymbol{\Lambda}_{i}(z)$ a paraunitary matrix of the form

$$
\boldsymbol{\Lambda}_{i}(z)=\mathbf{I}-\mathbf{v}_{i} \mathbf{v}_{i}^{\mathrm{H}}+z^{-\Delta_{i}} \mathbf{v}_{i} \mathbf{v}_{i}^{\mathrm{H}}
$$

with $\mathbf{v}_{i}=\left[\begin{array}{lllllll}0 & \cdots & 0 & 1 & 0 & \cdots & 0\end{array}\right]^{\mathrm{H}}$ containing zeros except for a unit element in the $\delta_{i}$ th position. Thus $\boldsymbol{\Lambda}_{i}(z)$ is an identity matrix with the $\delta_{i}$ th diagonal element replaced by a delay $z^{-\Delta_{i}}$.

At the $i$ th step, SBR2 will eliminate the largest off-diagonal element of the matrix $\tilde{\mathbf{B}}_{i-1}(z) \mathbf{R}_{1}(z) \mathbf{B}_{i-1}(z)$, which is defined by the two corresponding sub-channels and by a specific lag index. By delaying the two contributing sub-channels appropriately with respect to each other by selecting the position $\delta_{i}$ and the delay $\Delta_{i}$, the lag value is compensated. Thereafter a Givens rotation $\mathbf{Q}_{i}$ can eliminate the targetted element such that the resulting two terms on the main diagonal are ordered in size, leading to a diagonalisation and at the same time accomplishing a spectral majorisation.

Hence, each step comprises of optimising the parameter set $\left\{\delta_{i}, \Delta_{i}, \theta_{i}\right\}$. While the largest off-diagonal element in $\tilde{\mathbf{B}}_{i-1}(z) \mathbf{R}_{1}(z) \mathbf{B}_{i-1}(z)$ is eliminated, the remainder of the matrix is also affected. In extensive simulations, SBR2 has proven very robust and stable in achieving both a diagonalisation and spectral majorisation of any given covariance matrix, whereby the algorithm is stopped either after reaching a certain measure for suppressing off-diagonal terms or after exceeding a specified number of iteration $[2,6]$.

\subsection{Precoder and Equaliser for CCI Suppression}

The algorithm outlined above in Sec. 3.2 provides a factorisation $\mathbf{S}(z)=\tilde{\mathbf{B}}_{L}(z) \mathbf{H}(z) \mathbf{U}_{L}(z)$, whereby $\mathbf{S}(z)$ is approximately diagonalised, such that $\mathbf{U}_{L}(z)$ can be employed as a precoder and $\tilde{\mathbf{B}}_{L}(z)$ as an equaliser as shown in Fig. 1. Due to the decoupling, we can treat the resulting MIMO system $\mathbf{S}(z)$ as a system of separate subchannels. Due to spectral majorisation, the gain of the subchannels, and therefore their SNR, will drop with rising subchannel index. In the following we characterise this subchannel SNR.

Let $\mathrm{SNR}_{i}$ be the SNR of the $i$ th subchannel, $S_{i j}(z)$ the element of $\mathbf{S}(z)$ placed in the $i$ th row and $j$ th column, and $S_{i j}\left(e^{j \Omega}\right)$ its evaluation on the unit circle $z=e^{j \Omega}$. Further, $R_{x_{i}, x_{i}}(z)$ is the power spectral density of the $i$ th input in $\underline{X}(z)$, which we assume to be mutually uncorrelated with all other inputs. Then power spectral density of the signal component in the $i$ th channel, $R_{i}(z)$, is given by

$$
R_{i}(z)=S_{i i}(z) R_{x_{i}, x_{i}}(z) \tilde{S}_{i i}(z)
$$

The remaining CCI components can be characterised by the CCI power spectral density $R_{i, \mathrm{CCI}}(z)$,

$$
R_{i, \mathrm{CCI}}(z)=\sum_{j, j \neq i} S_{i j}(z) R_{x_{j}, x_{j}}(z) \tilde{S}_{i j}(z)
$$

The channel noise appears in the output $\underline{\hat{X}}(z)$ filtered by the equaliser $\mathbf{B}_{L}(z)$, such that is power spectral matrix at the output $\mathbf{R}_{e e}(z)=\mathbf{B}_{L}(z) \mathbf{R}_{v v}(z) \tilde{\mathbf{B}}_{L}(z)$. If the noise power spectral density $\mathbf{R}_{v v}(z)=\sigma_{v v}^{2} \mathbf{I}$, then due to paraunitarity of $\mathbf{B}_{L}(z)$, $\mathbf{R}_{e e}(z)=\sigma_{v v}^{2} \mathbf{I}$. Therefore, we obtain

$$
\mathrm{SNR}_{i}=\frac{1}{2 \pi} \int_{0}^{2 \pi} \frac{R_{i}\left(e^{j \Omega}\right)}{R_{i, \mathrm{CCI}}\left(e^{j \Omega}\right)+\sigma_{v v}^{2}} d \Omega
$$

The SNR value in (15) permits decisions on which subchannels are suitable for transmission, for example by means of waterfilling under the constraint of a given transmit power [4]. However, the problem of ISI within the selected subchannels remains, and will be addressed next.

\section{SISO Subchannel Precoding and Equalisation}

Various methods can be employed to eliminate the remaining ISI from the subchannels, such as the SISO precoding and equalisation approach according to [8]. There, jointly optimal transmitter-receiver filter bank pairs for block transmission over frequency selective channels in the presence of noise are derived. 


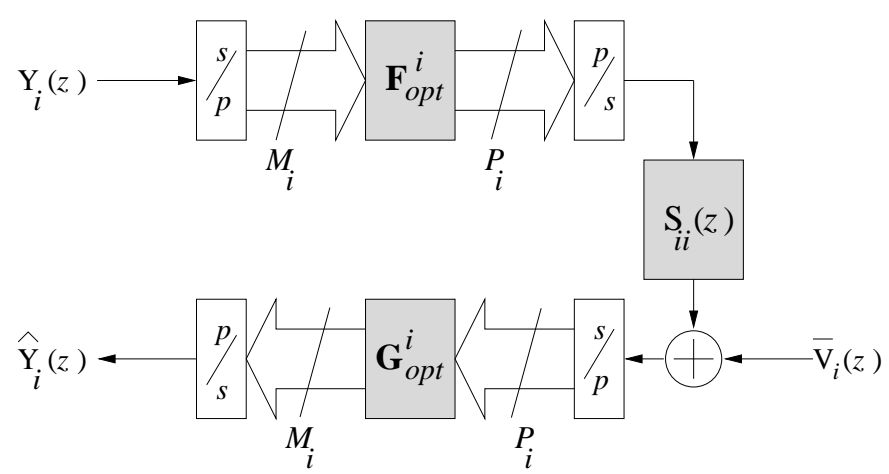

Fig. 2. Joint precoding and equalization for frequency selective SISO channel.

Our explanations are based on the precoding and equalisation architecture provided in Fig. 2, which only shows the arrangement for the $i$ th subchannel. We define $\mathbf{R}_{y_{i} y_{i}}$ as the covariance matrix of the input signal $Y_{i}(z)$ and $\mathbf{R}_{\bar{v}_{i} \bar{v}_{i}}$ as the noise covariance matrix arising from the signal $\bar{V}_{i}(z)$. Note that the latter comprises of the channel noise filtered by $\tilde{\mathbf{B}}(z)$ and the CCI components of the $i$ th channel characterised in (14), such that at the input of the equaliser $\mathbf{G}_{\mathrm{opt}}^{i}$ we measure the SNR stated in (15). Further, the Toeplitz matrix $\mathbf{S}^{(i)}$ contains the impulse response of length $L_{i}$ of the $i$ th subchannel, $S_{i i}(z)$, produced by the BSVD in Sec. 3.3,

$$
\mathbf{S}_{(i)}=\left[\begin{array}{cccc}
s^{(i)}[0] & 0 & \cdots & 0 \\
\vdots & \ddots & \ddots & \vdots \\
s^{(i)}\left[L_{i}-1\right] & & \ddots & 0 \\
0 & \ddots & & s^{(i)}[0] \\
\vdots & \ddots & \ddots & \vdots \\
0 & \cdots & 0 & s^{(i)}\left[L_{i}-1\right]
\end{array}\right]
$$

whereby $S_{i i}(z)=\sum_{n=0}^{L_{i}-1} s^{(i)}[n] z^{-n}$. The use of a single time slice as opposed to the analysis in (4) stems from the block based transmission avoiding inter-block interference by means of either $L_{i}$ leading zeros or $L_{i}$ discarded symbols at the receiver, thus requiring $P_{i} \geq M_{i}+L_{i}$. Based on $\mathbf{R}_{y_{i} y_{i}}, \mathbf{R}_{\bar{v}_{i} \bar{v}_{i}}$, and $\mathbf{S}_{(i)}$, we define the following EVD factorisations:

$$
\begin{aligned}
\mathbf{R}_{y_{i} y_{i}} & =\mathbf{W}_{i} \boldsymbol{\Delta}_{i} \mathbf{W}_{i}^{\mathrm{H}} \\
\mathbf{S}_{(i)}^{\mathrm{H}} \mathbf{R}_{\bar{v}_{i} \bar{v}_{i}}^{-1} \mathbf{S}_{(i)} & =\mathbf{V}_{i} \boldsymbol{\Lambda}_{i} \mathbf{V}_{i}
\end{aligned}
$$

with the diagonal matrices

$$
\begin{aligned}
& \boldsymbol{\Delta}_{i}=\operatorname{diag}\left\{\delta_{00}^{(i)}, \delta_{11}^{(i)}, \cdots \delta_{M_{i}-1, M_{i}-1}^{(i)}\right\} \\
& \boldsymbol{\Lambda}_{i}=\operatorname{diag}\left\{\lambda_{00}^{(i)}, \lambda_{11}^{(i)}, \cdots \lambda_{M_{i}-1, M_{i}-1}^{(i)}\right\}
\end{aligned}
$$

The method outline in [8] proposes the design of $\mathbf{G}_{\mathrm{opt}}^{i}$ and $\mathbf{F}_{\text {opt }}^{i}$ under two criteria, either maximising the output SNR (max-SNR) or minimising the mean-square error under con- strained transmit power (MMSE/CP). In the first case, the maxSNR optimum filter bank pair is given by

$$
\begin{aligned}
\mathbf{F}_{\mathrm{opt}}^{i} & =\frac{\sqrt{K}}{\sigma_{\bar{v}_{i}}} \mathbf{V}_{i} \boldsymbol{\Lambda}_{i}^{-\frac{1}{2}} \\
\mathbf{G}_{\mathrm{opt}}^{i} & =\sigma_{\bar{v}_{i}} \sqrt{K} \boldsymbol{\Lambda}^{-\frac{1}{2}} \mathbf{V}_{i}^{\mathrm{H}} \mathbf{S}_{(i)}^{\mathrm{H}} \mathbf{R}_{\bar{v}_{i} \bar{v}_{i}}^{-1}
\end{aligned}
$$

where $K$ is gain in the transmitter. The MMSE/CP optimum filter bank pair is given by

$$
\begin{aligned}
\mathbf{F}_{\mathrm{opt}}^{i} & =\mathbf{V}_{i} \boldsymbol{\Phi}_{i} \mathbf{W}_{i}^{\mathrm{H}} \\
\mathbf{G}_{\mathrm{opt}}^{i} & =\frac{\mathbf{R}_{y_{i} y_{i}}\left(\mathbf{F}_{\mathrm{opt}}^{i}\right)^{\mathrm{H}} \mathbf{S}_{(i)}^{H}}{\mathbf{R}_{\bar{v}_{i} \bar{v}_{i}}+\mathbf{S}_{(i)} \mathbf{F}_{\mathrm{opt}}^{i} \mathbf{R}_{y_{i} y_{i}}\left(\mathbf{F}_{\mathrm{opt}}^{i}\right)^{\mathrm{H}} \mathbf{S}_{(i)}^{H}}
\end{aligned}
$$

where $\boldsymbol{\Phi}_{i}$ is diagonal with elements given by

$$
\left|\phi_{j j}^{(i)}\right|^{2}=\frac{P_{i}+\operatorname{tr}\left\{\boldsymbol{\Lambda}_{i}^{-1}\right\}}{\operatorname{tr}\left\{\boldsymbol{\Lambda}_{i}^{-\frac{1}{2}} \boldsymbol{\Delta}_{i}^{\frac{1}{2}}\right\}} \frac{1}{\sqrt{\lambda_{j j}^{(i)} \delta_{j j}^{(i)}}}-\frac{1}{\lambda_{j j}^{(i)} \delta_{j j}^{(i)}}
$$

in order to enforce the constraining of the transmit power of the $i$ th subchannel to $P_{i}$ [8].

\section{Simulations and Results}

\subsection{Simulation}

In this section, we illustrate the proposed approach by means of a numerical example. We assume a $4 \times 4$ broadband MIMO channel whose temporal responses are generated from a SalehValenzuela indoor channel model [10]. The resulting MIMO channel length is $L=5$.

The diagonalisation and spectral majorisation of $\mathbf{H}(z)$ leads to 4 subchannels with frequency responses ordered in descending value, as shown in Fig. 3. It is clear that of the 4 subchannels, only the first three offer a reasonable channel gain and therefore sufficient quality for transmission, thus setting $M=3$ or potentially lower.

For each subchannel, a precoder and equaliser is computed according to Sec. 4. With subchannel lengths of $L_{i} \in\{56 ; 63\}$ and $i=0,1, \cdots \quad M-1$, a block size of $M_{i}=32$ is employed for all $M$ systems for simplicity. In order to assess the result of the design process, we measure the BER in terms of an SNR figure suggested in [8], which sets the afforded transmit power against the channel noise measured at the SISO receiver. Thus, in this case $\mathrm{CCI}$ is considered as a channel noise component, such that for the $i$ th subchannel

$$
\mathrm{SNR}=\frac{\operatorname{tr}\left\{\mathbf{F}_{\mathrm{opt}}^{i}\left(\mathbf{F}_{\mathrm{opt}}^{i}\right)^{\mathrm{H}}\right\}}{R \sigma_{\bar{v}_{i}}^{2}} .
$$

It can clearly be noted from Fig. 4 that the three selected subchannels offer a degrading BER performance with rising subchannel index.

The further exploitation of the system depends on the selection of the number of subchannels utilised for transmission, as well as the way in which the overall available power $P$ is spent amongst the selected subchannels. Both selection and power distribution can be solved by waterfilling methods [4], which are not further elaborated here. 


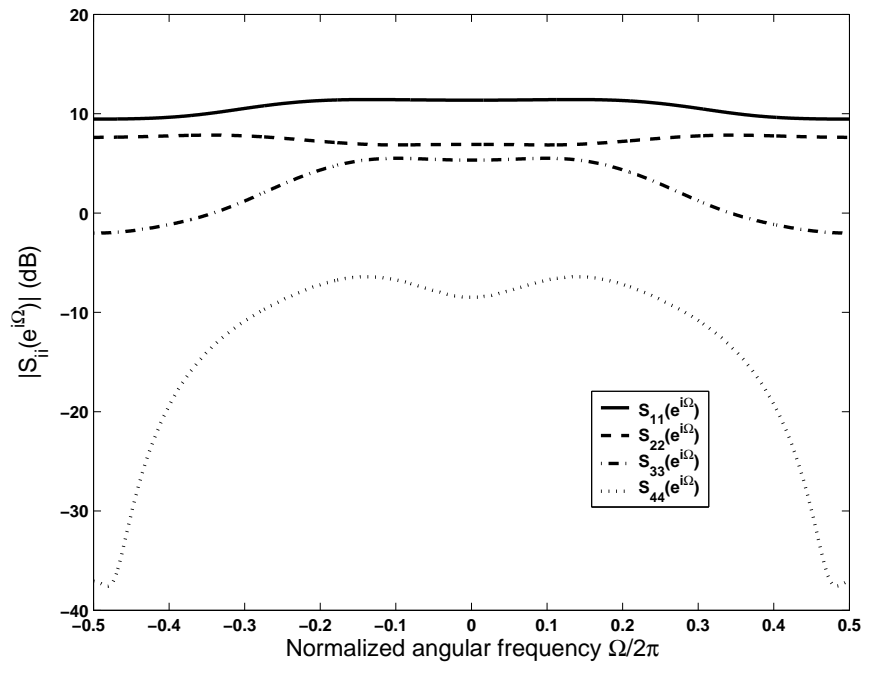

Fig. 3. Frequency responses $S_{i i}\left(e^{j \Omega}\right)$ of subchannels after diagonalisation of $\mathbf{H}(z)$.

\section{Conclusion}

We have discussed a design of precoders and equalisers for broadband MIMO systems which are based on two separate steps. First, the CCI imposed by the MIMO transmission system is suppressed by means of a BSVD, similar to how a standard SVD would be employed for a narrowband MIMO channel. Second, SISO precoding and equalisation techniques are invoked in order to mitigate ISI within the subchannels.

The proposed scheme differs from other works, e.g. [8, 9], in the absence of a block transmission scheme for the first step, i.e. the CCI cancellation. The use of the SBR2 algorithm to accomplish a BSVD has some pitfalls since it allows little control over the filter order that is obtained for the diagonalised system and therefore the decoupled subchannels. Note however that in Fig. 3 the the first two subchannels exhibit only very low spectral dynamics and would therefore only require an equaliser (and precoder) of relatively small order. Therefore, future work will attempt to further exploit the potentials of this approach.

\section{Acknowledgements}

The research of Chi Hieu Ta is supported by a scholarship from the Vietnamese Ministry of Education and Training.

\section{References}

[1] A. Goldsmith, S. A. Jafar, N. Jindal, and S. Vishwanath. Capacity Limits of MIMO Channels. IEEE JSA, 21(5): , 2003.

[2] J. G. McWhirter and P. D. Baxter. A Novel Technqiue for Broadband SVD. In Workshop Adaptive Sensor Array Proc., Cambridge, MA, 2004.

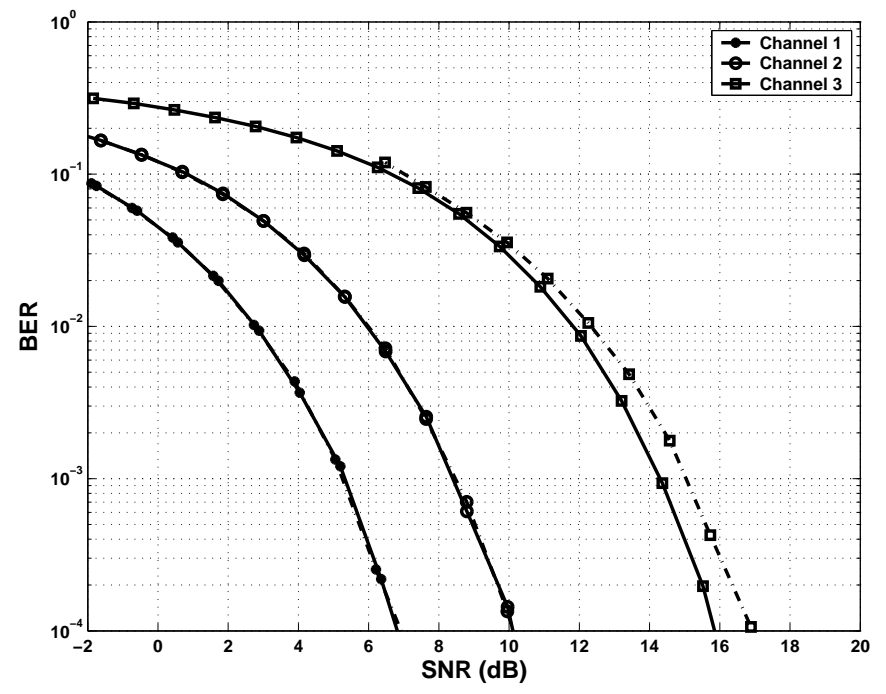

Fig. 4. BER versus SNR for the subchannels $S_{i i}\left(e^{j \Omega}\right), i=$ $0,1,2$ after SISO precoding and equalisation according to [8].

[3] D. P. Palomar, J. M. Cioffi, and M. A. Lagunas. Joint TxRx Beamforming Design for Multicarrier MIMO Channels: A Unified Framework for Convex Optimization. IEEE Trans SP, 51(9):2381-2401, 2003.

[4] D. P. Palomar and J. R. Fonollosa. Practical Algorithms for a Family of Waterfilling Solutions. IEEE Trans SP, 53(2):686-695, 2005.

[5] C. Peel, Q. Spencer, A. L. Swindlehurst, and B. Hochwald. Downlink Transmit Beamforming in Multi-User MIMO Systems. In IEEE Workshop Sensor Array and Multichannel Signal Proc., vol. 1, Sitges, Spain, 2004.

[6] S. Redif and T. Cooper. Paraunitary Filter Bank Design via a Polynomial Singular Value Decomposition. In Proc. IEEE ICASSP, 4: 613-616, Philadelphia, PA, 2005.

[7] A. Scaglione, G. B. Giannakis, and S. Barbarossa. Filterbank Transceivers Optimizing Information Rate in Block Transmission over Dispersive Channels. IEEE Trans Inf. Theory, 45(4):1019-1032, 1999.

[8] A. Scaglione, G. B. Giannakis, and S. Barbarossa. Redundant Filterbank Precoders and Equalizers. I. Unification and Optimal Designs. IEEE Trans SP, 47(7):1988-2006, 1999.

[9] A. Scaglione, P. Stoica, S. Barbarossa, G. B. Giannakis, and H. Sampath. Optimal Designs for Space-Time Linear Precoders and Decoders. IEEE Trans SP, 50(5):10511064, 2002.

[10] A.A.M Saleh and R.A. Valenzuela. A Statistical Model for Indoor Multipath Propagation. IEEE JSAC, 5(2):128137, 1997.

[11] P. P. Vaidyanathan. Theory of Optimal Orthonormal Subband Coders. IEEE Trans SP, 46(6):1528-1543, 1998. 